\title{
CAN Communication Interface Design Based On TMS320F2812
}

\author{
Na Fang ${ }^{1, a}$, Hanlin Huang ${ }^{2, b}$, Zhimin Deng ${ }^{2, b}$, Yunbo Zhu ${ }^{1, a}$ \\ ${ }^{1}$ Wuhan Mechanical Technology College, Wuhan, 430075,China \\ ${ }^{2}$ Military Representative Office in Wuhan, Xiangyang, 441813, China \\ aE-mail: 641573862@qq.com, ${ }^{\mathrm{b} E}$-mail: 28656805@qq.com
}

Keywords: CAN; TMS320F2812; Center-control Computer; Transceiver; Optical Isolation.

\begin{abstract}
The article presents a design of CAN(Controller Area Network) communication system based on DSP TMS320F2812. It introduces the hardware circuit and software design methods in detail. The schematic diagram of hardware is shown as well as the program diagram of system receiving and sending data. This system implements the data communication between servo system and center-control computer. At the same time, the speed of communication and the antiinterference ability are improved.
\end{abstract}

\section{Introduction}

The currently existing communication between servo systems and center-control computer is RS232 serial connection for the most part. The voltage of each signal line is referenced with ground voltage because of the three-wire communication style of RS232.The discrepant voltage to ground between sender and receiver in transmission could easily bring about a communication error or speed reduction and anti-interference ability is poor[1]. Compared with RS232, CAN communication uses a two-wire differential means of communication and its data transmission is short frame transmission[2]. Therefore, CAN communicates in real time, high reliability, strong anti-interference ability, long distance transmission, fast communication and so on[3].

CAN communication is utilized in the modes of communication between servo system and center-control computer in this article. TMS320F2812, the DSP controller built-in CAN is used in the software and hardware design of CAN communication system[4].

\section{System Configuration}

The schematic diagram of CAN communication system is shown in Figure 1.The system is composed of fire-control computer, DSP, CAN transceiver, optical isolators. DSP is the main controller of servo system, choosing TMS320F2812 of TI Company. CANTX is CAN's sending data cable and CANRX is receiving data cable.

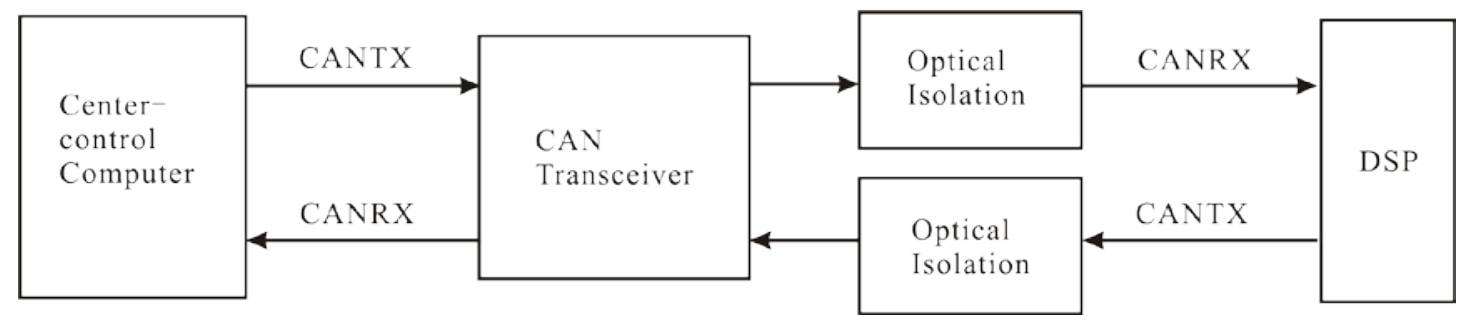

Fig.1. Schematic Diagram of CAN Communication System. 


\section{System Design}

\section{A. CAN Transceiver Design}

CAN transceiver is the driving connector of CAN controller and physical bus, providing differential transmitting and receiving function to the bus. SN65HVD251 of TI Company is used for CAN transceiver in this system, and line interference is employed. It can provide over-voltage, $\pm 36 \mathrm{~V}$ and thermal shutdown protection. In addition, it can work sustainably with high common mode and impedance in a disconnected state [5]. And it is fully compatible with DSP TMS320F2812. Its schematic diagram is shown in Figure 2.

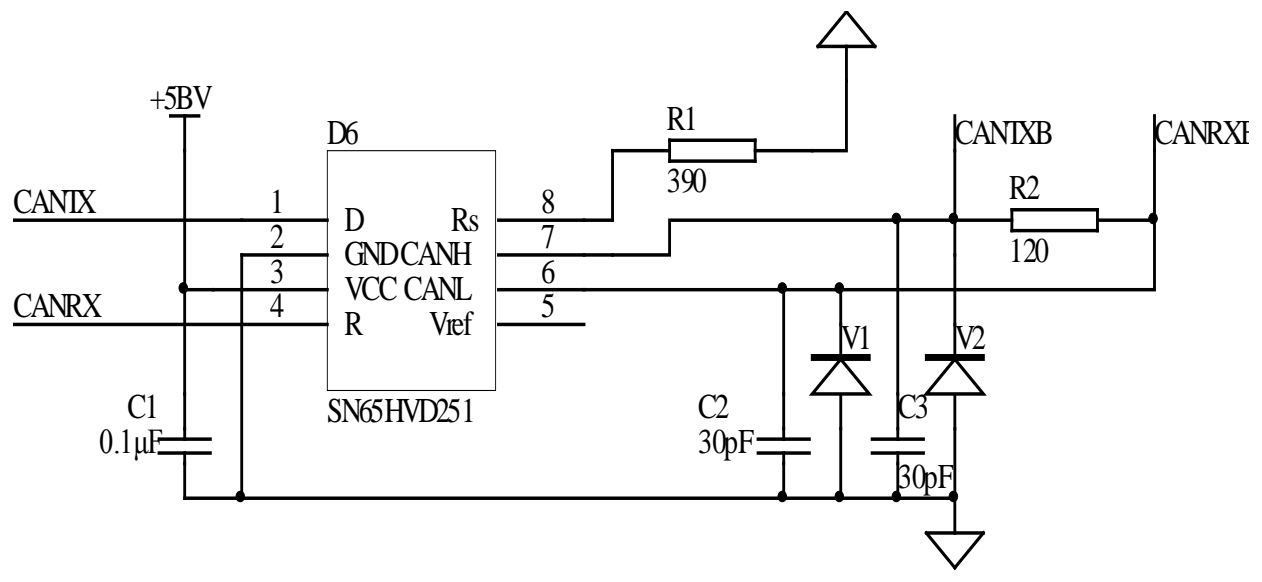

Fig.2. Schematic Diagram of CAN Transceiver.

In this schematic diagram, resistor R3 plays a very important role for bus impedance matching. Ignoring it will make data communication anti-jamming and reliability of greatly reducing or even incapable communications. Resistor R1 is called the slope resistance, which determines the system working in high speed mode or the slope-control mode [5]. Directly connecting the PIN with ground, the system will be work in high speed mode. In slope-control mode, the resistance of resistor R1 is associated with the speed of communication. The smaller the resistance is, the faster the speed is.

\section{B. Optical Isolation Design}

Inevitably, there are all kinds of interference signals in an actual circuit system. In order to strengthen the anti-interference ability of CAN communication, optoelectronic isolating circuit is designed in the system[6]. It can effectively prevent jammer's entering, and ensure signal isolation reliably. At the same time, it can reduce the interference effect on the system, and improve the reliability of CAN communication. Its schematic diagram is shown in Figure 3.

The operating voltage of optical couplers D4, D5 is 5-volt, which is consisted of two separate power supply module. And optical couplers D4, D5 have no common ground. In addition, CANTXA is connected with the CAN data transmission of TMS320F2812. Therefore, the import reference voltage of D4 is 3.3 volts. Likewise, the import reference voltage of D5 is 5 volts.

\section{Software Design}

Before trying to use the mailbox of CAN module of TMS320F2812 controller, we must execute the parameter settings as necessary, develop communication baud rate and set the identifier as well as a number of control bits. The corresponding mailboxes are configured according to the different ways of accessing data registers. 


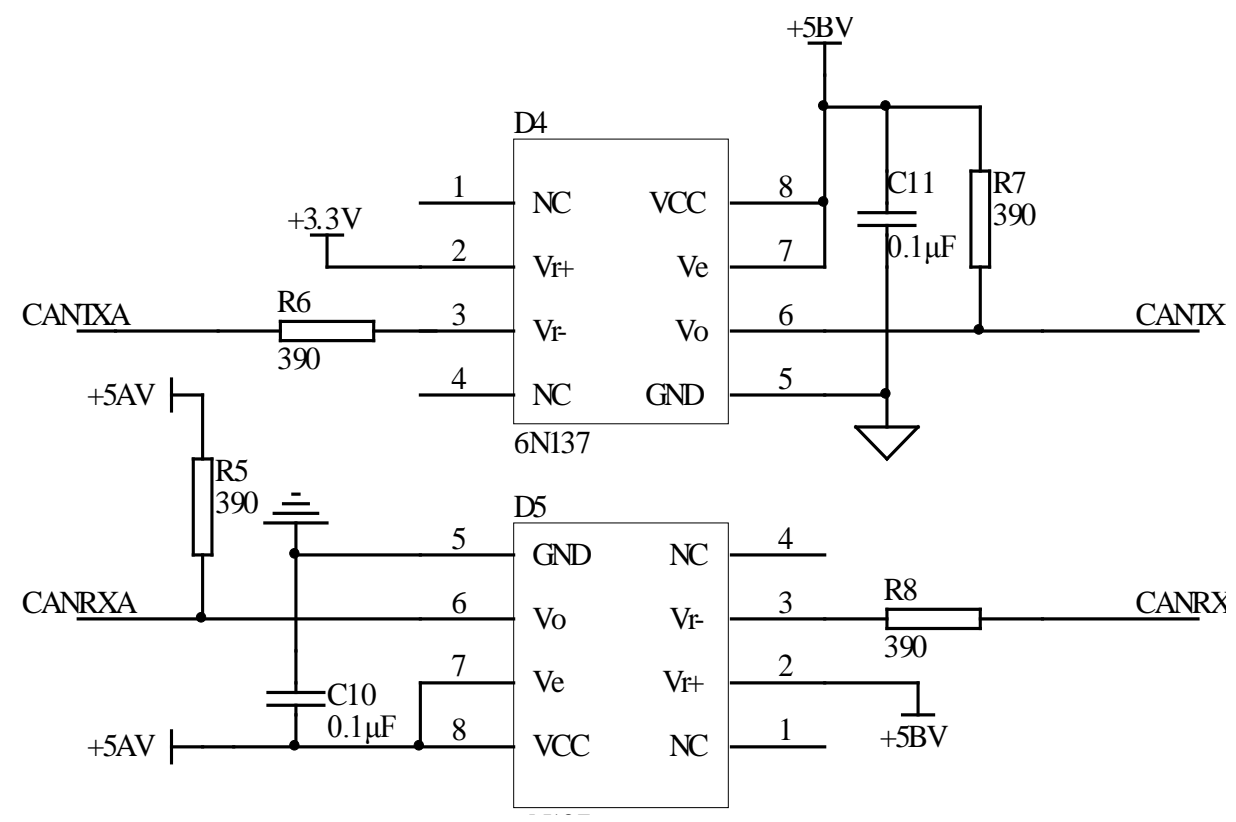

Fig.3. Schematic Diagram of Optical Isolation.

\section{A. CAN Module Parameters Configuration}

I/O Port: Because CAN addresses the two pins for CANTX and CANRX multiplexing I/O port, the first step of CAN communication software programming is setting GPIO6 and GPIO7 of TMS320F2812 to 1 respectively.

Initialize the digit timer: digit timer is mainly consisted of BCR1 register and BCR2 register, including communication baud rate, synchronization jump width, sampling frequency and synchronization methods of CAN controller [7]. The external clock frequency of this system is $30 \mathrm{MHz}$. The PLL frequency coefficients is 0x000A.The system frequency is $150 \mathrm{MHz}$. The communication baud rate is $500 \mathrm{~K}$.

Initialize the mailbox: mailbox initialization is mainly setting up the identifier and control domain of mailbox (a remote frame or a data frame), as well as assigning initial values to corresponding mailbox.

\section{B. Data Transmittal}

When module parameter configuration of CAN is finished, we can send and receive data. The flow diagram of data transmittal is shown in Figure 4.

\section{Data Reception}

The data can be accepted only when the received message identifiers must be identical to the corresponding received mailbox. Otherwise, the information is filtered. We can use the query method for the data incoming requests here. The flow diagram of data reception is shown in Figure 5.

\section{Conclusion} the

Because of the CAN communication system which is constituted by TMS320F2812 controller, 


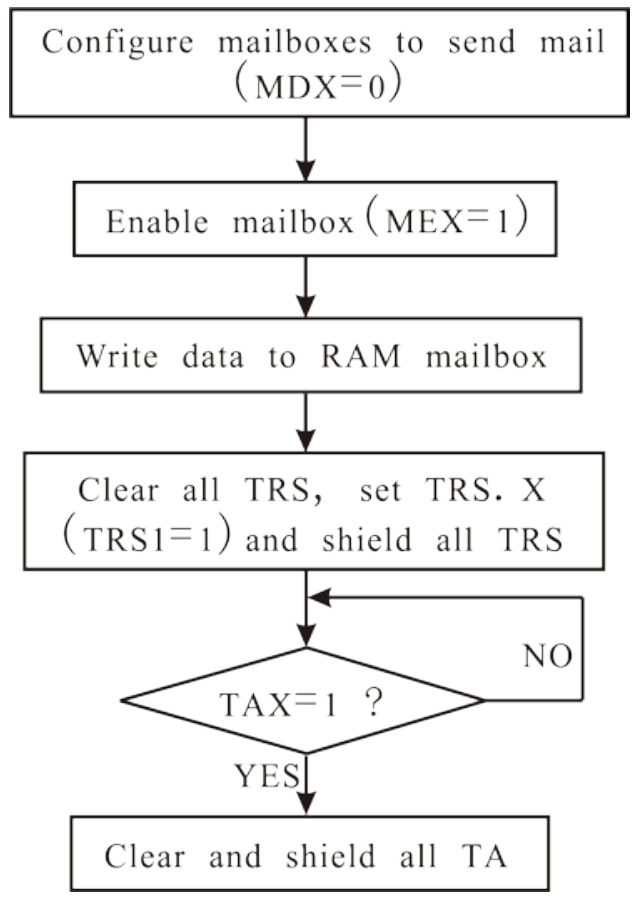

Fig.4. The Flow Diagram of Data Transmittal.

Communication between center-control computer and servo system becomes more reliable. The speed of communication is improved. The anti-interference ability is strengthened. In addition, CAN, which is embedded in TMS320F2812, makes hardware design of the system simpler and software realization easier.

\section{References}

[1]Wu Kuanming. Principle And Application Of CAN Bus System Design[M]. Beijing University Of Aeronautics And Astronautics Press, 1996, pp.91.

[2]Rao Yuntao, Zou Jijun and Zheng Yongyun. Principle And Application Of Field Bus CAN[M]. Beijing University Of Aeronautics And Astronautics Press, 2003, pp.116.

[3] Li Zhengjun. Field Bus And Its Application[M]. Mechanical Industry Publishing House, 1996, pp.129.

[4]Su Kuifeng. TMS320F2812 Principle And Development [M]. Beijing: Publishing House of Electronics Industry, 2005.04, pp.70-72.

[5]The High Speed CAN Transceiver 5V SN65HVD251. Electronic Components, 2003.03, pp.78.

[6] Liu Fahui. CAN Communication System Design Based On SJA1000 [J]. Field Bus And Network Technologies, 2006.01, pp.72-74.

[7] Wang Yunwu, Wei Zongshou. Design Communication Based On CAN Bus Node [J]. Mod-ern Electronic Technology, 2008.3. 